\title{
The Neuroprotective Role of Progesterone in Traumatic Brain Injury; Reduction of Inflammatory Cytokines
}

\section{Sajad Sahab Negah ${ }^{1,2}$, Arezou Eshaghabadi', Elham Mohammadzadeh ${ }^{1,3^{*}}$ \\ ${ }^{1}$ Shefa Neuroscience Research Center, Khatam Alanbia Hospital, Tehran, Iran}

${ }^{2}$ Histology and Embryology Group, Basic Science Department, Faculty of Veterinary Medicine, Shiraz University, Shiraz, Iran ${ }^{3}$ Department of Biology and Anatomical Sciences, Faculty of Medicine, Shahid Beheshti University of Medical Sciences, Tehran, Iran

\section{ABSTRACT}

Introduction: Traumatic brain injury (TBI) is one of the leading causes of death of men under the age of 35 that mostly occurs during the road accidents. Current clinical treatments cannot prevent the secondary brain damage induced by cytotoxicity. Progesterone, as a primary sex hormone, is a neuroprotective compound in TBI and stroke. Indeed, progesterone increases anti-oxidant activities, decreases inflammatory factors, and reduces the expression of inflammatory cytokine, which can prevent secondary brain injury and edema. Conclusion: This review focused on the preventive effect of progesterone on secondary brain injury and edema by modulation of inflammatory factors in TBI. Although, the exact mechanism of TBI has not been discovered, studies suggest that this hormone can be a safe treatment for TBI and other neurodegenerative diseases.

* Corresponding Author: Elham Mohammadzadeh

E-mail: elhammohammadzadeh85@gmail.com

\section{Key words:}

1. Brain Injuries

2. Progesterone

3. Neuroprotective Agents 


\title{
نقش حفاظت عصبى يروزسترون در آسيب مغزى يس از ضربه؛ كاهش سايتوكينهاى التهابى
}

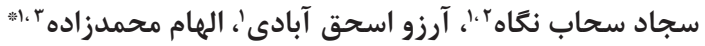 \\ 'مركز تحقيقات علوم اعصاب شفا، بيمارستان خاتم الانبياء، تهران، ايران \\ 'بخش بافتشناسى و جنينشناسى، كروه علوم يايه، دانشكده داميزشكى، دانشكاه شيراز، شيراز، ايران \\ "كَروه بيولوزى و علوم تشريح، دانشكده يزشكى، دانشخاه علوم يزشكى شهيد بهشتى، تهران، ايران
}
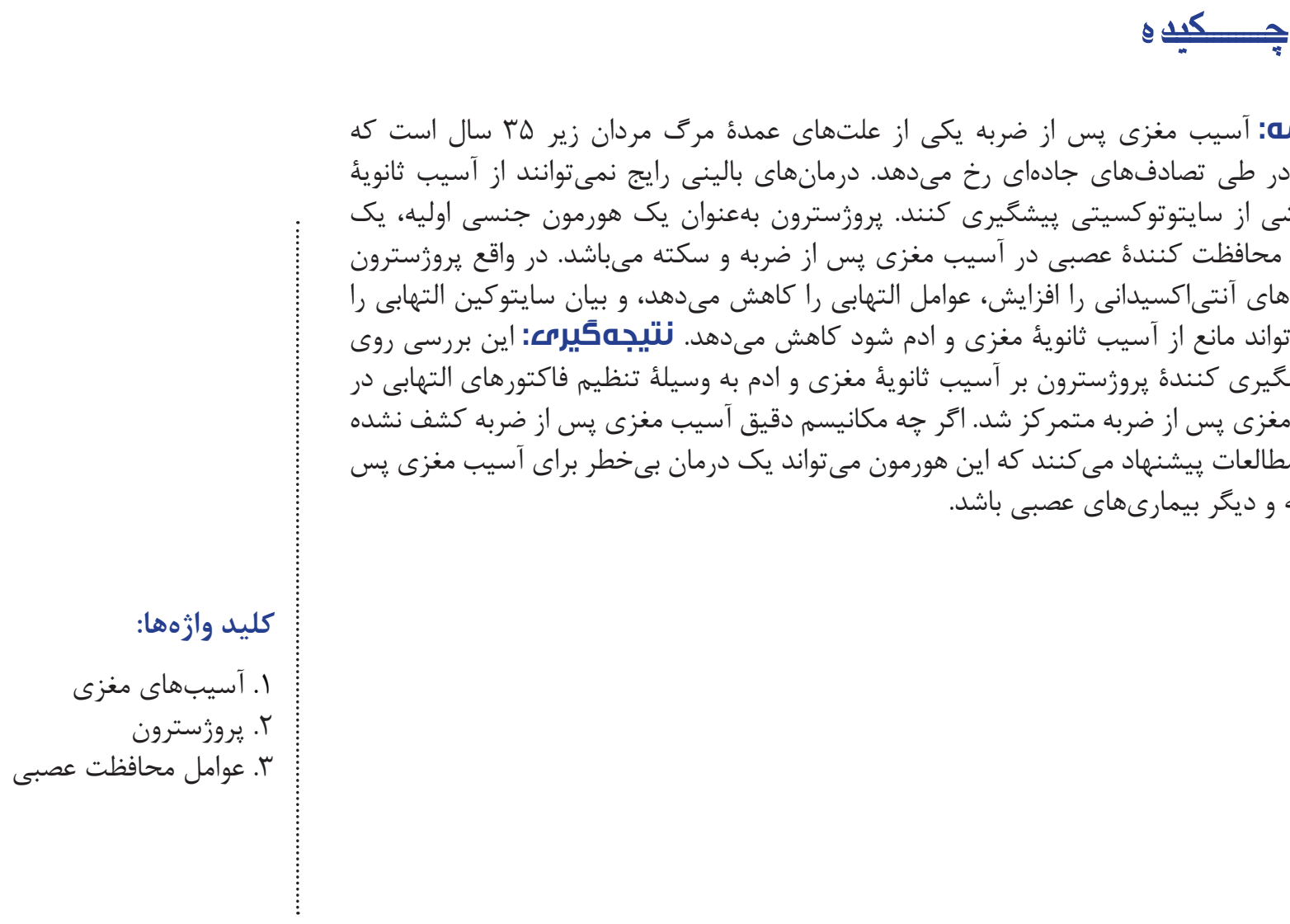

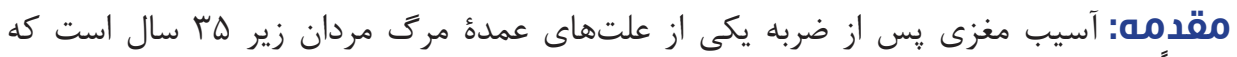

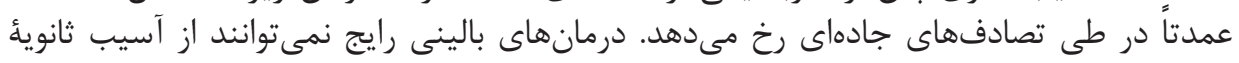

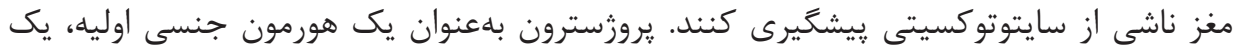

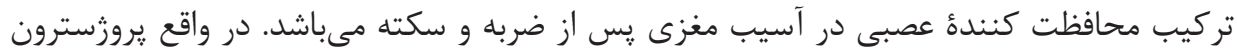

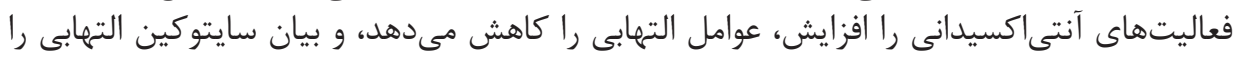

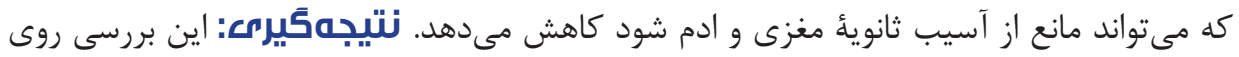

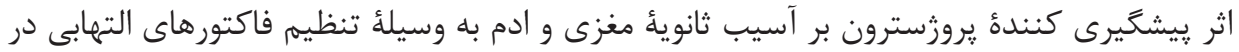

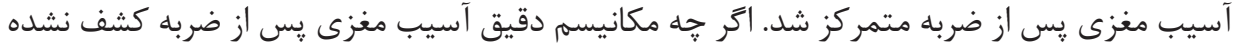

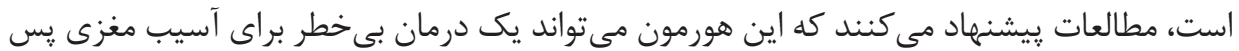
از ضربه و ديكر بيمارىهاى عصبى باشد.

" نويسنده مسئول: الهام محمدزاده آدرس الكترونيكى: elhammohammadzadeh85@gmail.com 


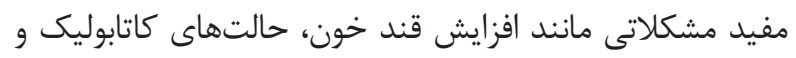

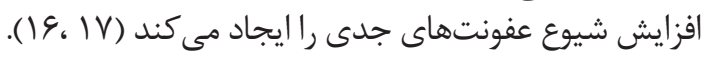

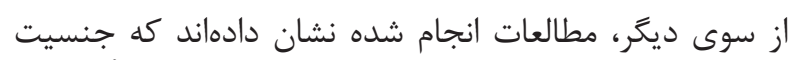

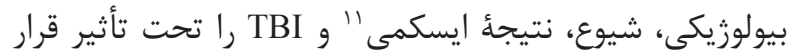

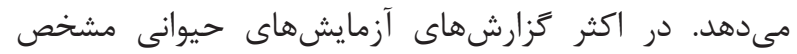

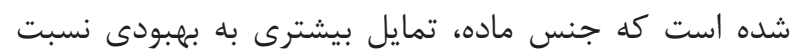

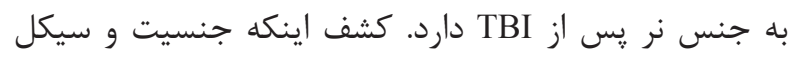

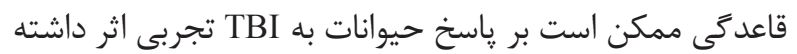

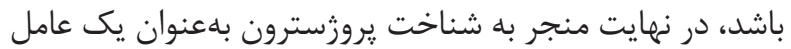

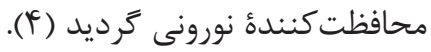

يروزسترون يك هورمون استروئيدى است كه به دليل نقش تش آن در

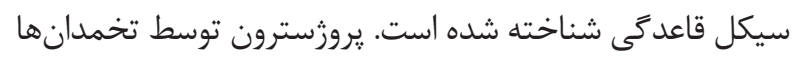

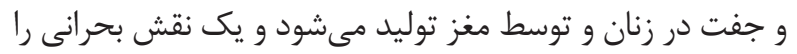

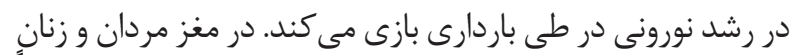

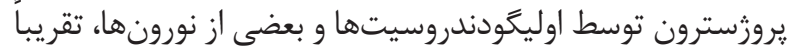

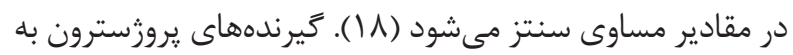

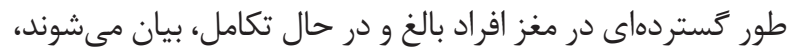

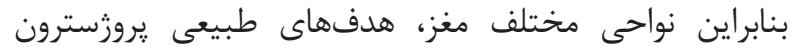

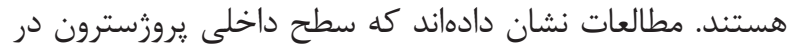

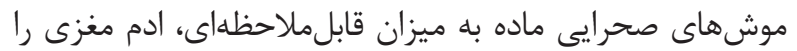

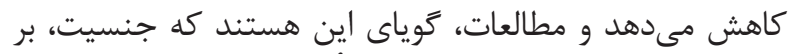

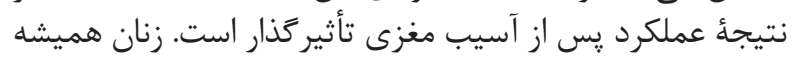

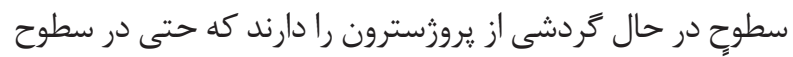

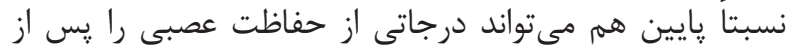

آسيب مغزى موضعى، سبب شود (؟).

در واقع يروزسترون نهتنها بهعنوان يك هورمون توليدمثلى ماده

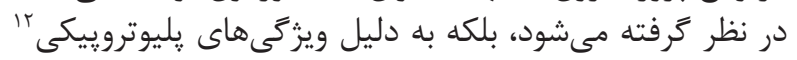

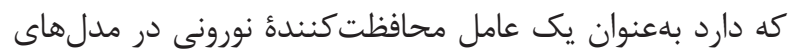

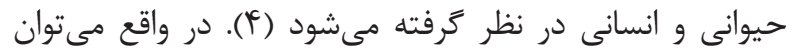

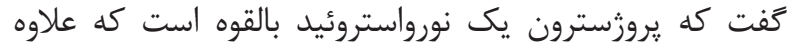

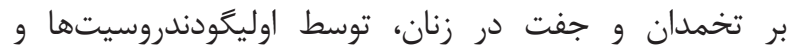

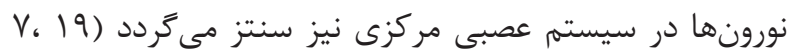

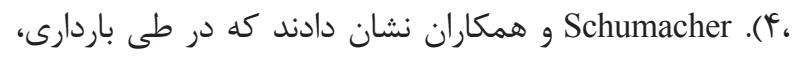

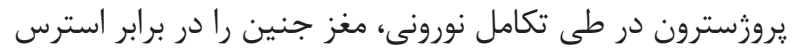

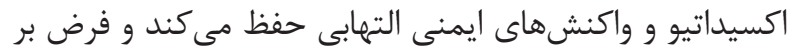

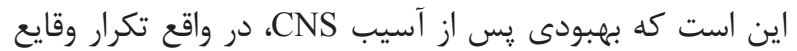

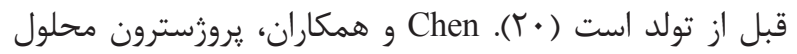

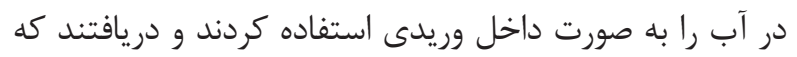

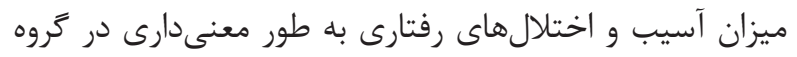

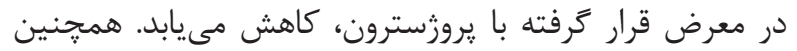

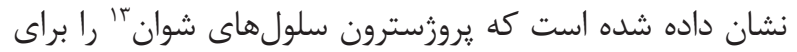

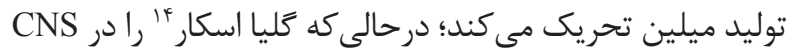

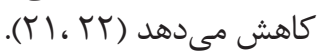

${ }^{1}$ Traumatic brain injury

${ }^{2}$ Hypertension

${ }^{3}$ Brain herniation

${ }^{4}$ Mannitol

${ }^{5}$ Barbiturates

${ }^{6}$ Corticosteroids

${ }^{7}$ Hyperventilation

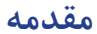

آسيب مغزى پِّ از ضربه (TBI)' علت اصلى مركى و ناتوانى شديد در سراسر جهان است؛ از علل اصلى آن آن مى آتوان به به تصادفات

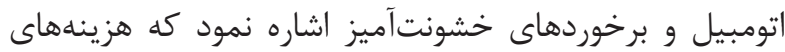

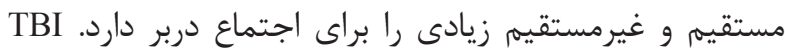

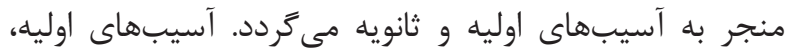

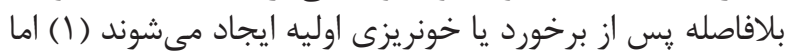

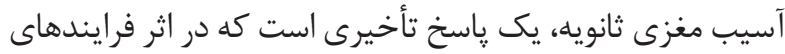

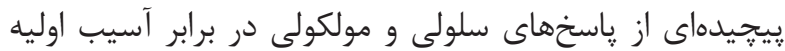

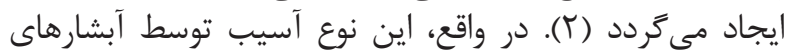

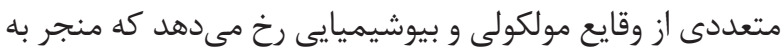

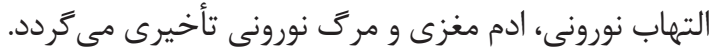

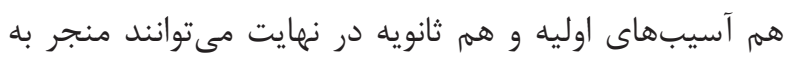

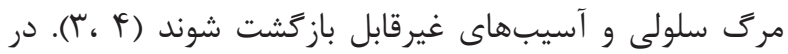

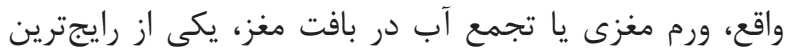

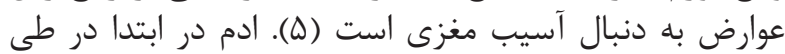

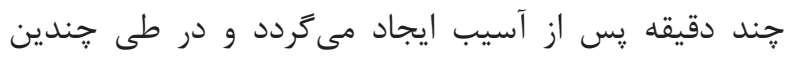

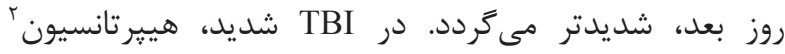

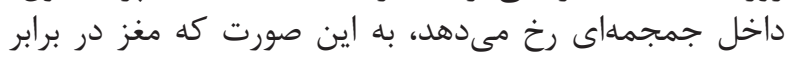

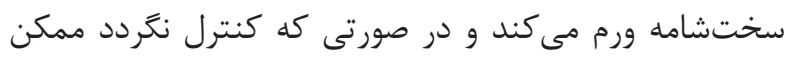

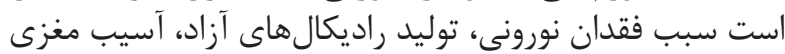

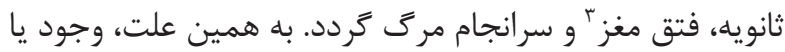

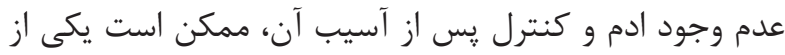

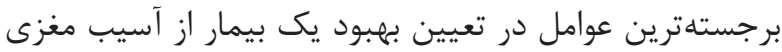

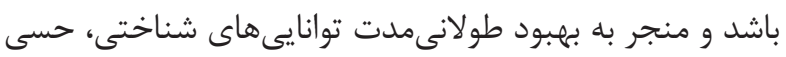

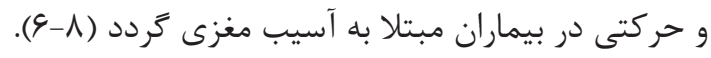

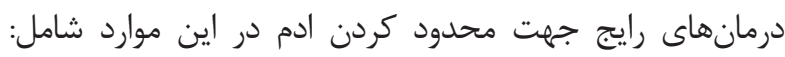

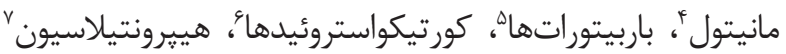

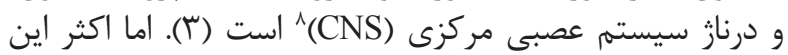

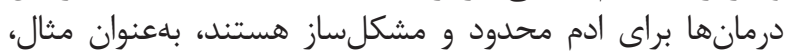

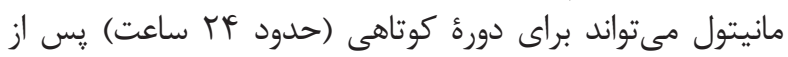

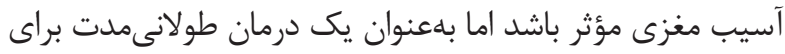

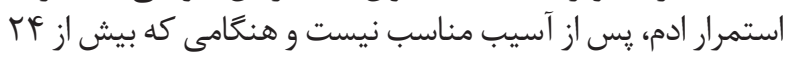

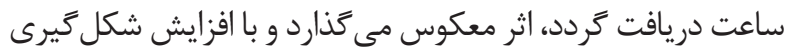

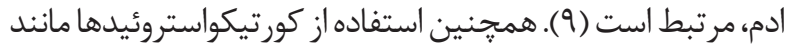

متيل يردنيزولون" با درمان هاى محدودى روبرو شده است (• (1).

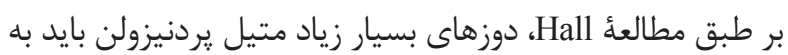

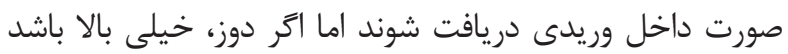

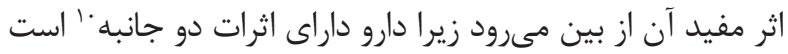

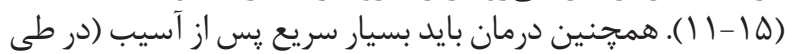

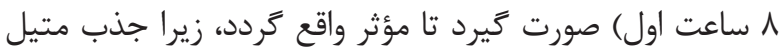

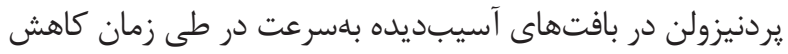
مى يابد. از سوى ديخر، مقادير مورد نياز براى به دست درئ آوردن نتايج كاهث

${ }^{8}$ Central nervous system

${ }^{9}$ Methylprednisolone

${ }^{10}$ Biphasic

${ }^{11}$ Ischemia

${ }^{12}$ Pleiotropic

${ }^{13}$ Schwann cells

${ }^{14}$ Glia scar 
دادن يكيارجكى غشاء، بسيارى از مسيرها را تحريك مى كند

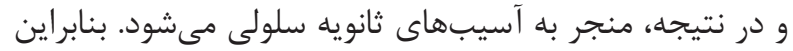

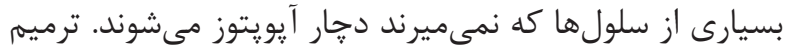

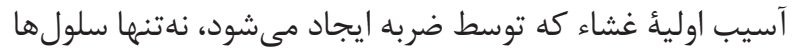

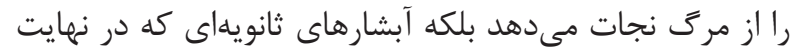

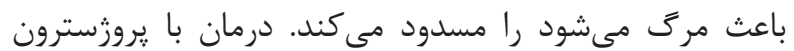

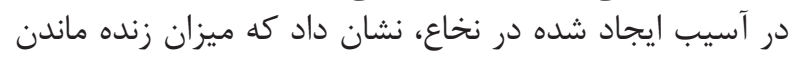

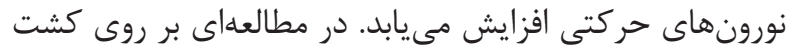

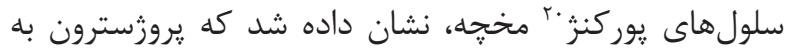
طور غيرمستقيم از طريق فعاليت كيرندة

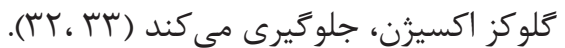

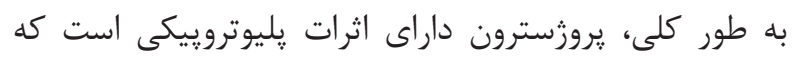

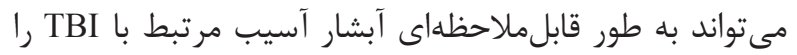

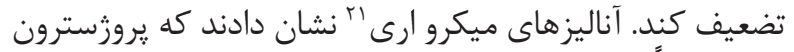

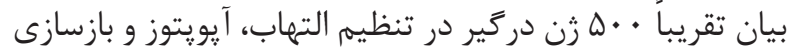

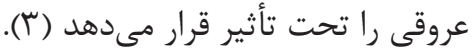

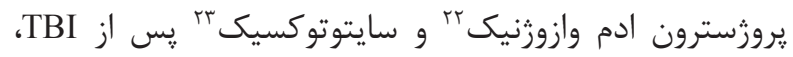

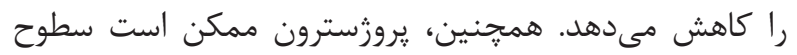

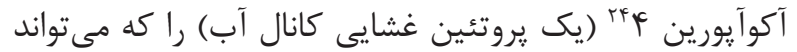

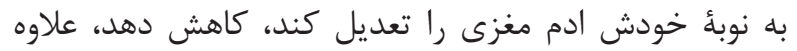
بر اين جذب فعال يونى را از طريق ران تعديق

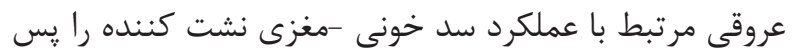

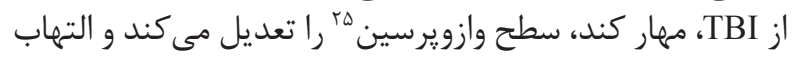

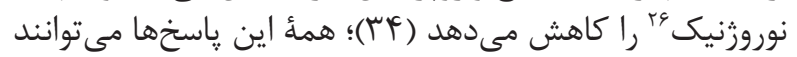

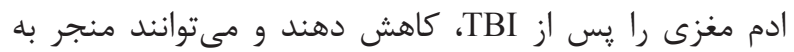

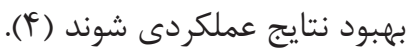

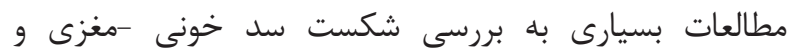

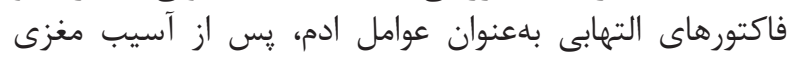

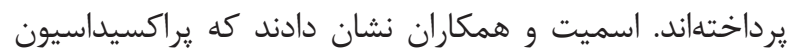

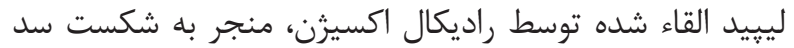

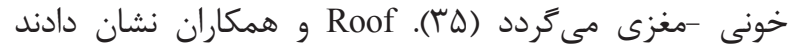

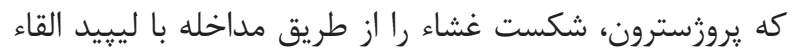

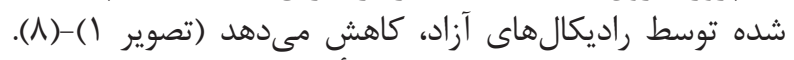

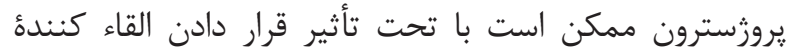

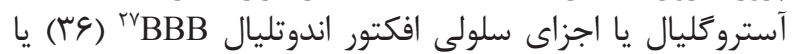

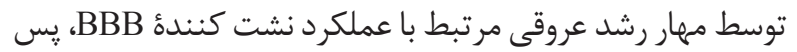

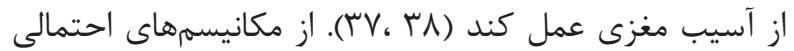

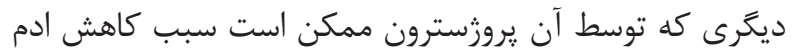

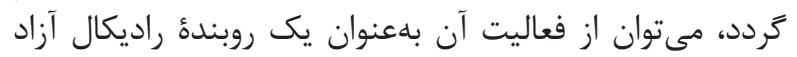

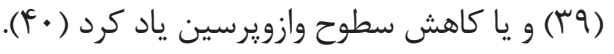
همجنين تعدادى از مطالعات نشان دادهاند كه اثر محافظتى

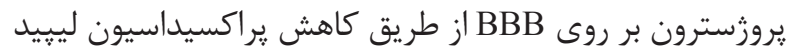

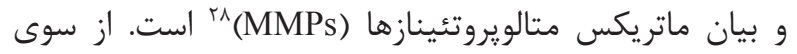

\footnotetext{
${ }^{15}$ Neurosteroid

${ }^{16}$ Signaling

${ }^{17}$ gamma-Aminobutyric acid A

${ }^{18}$ Allopregnanolone

${ }^{19}$ Lactate dehydrogenase

${ }^{20}$ Purkinje cells

${ }^{21}$ Microarray
}

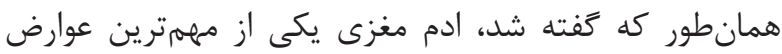
جانبى آسيبهاى مغزى ترون تروماتيك است.

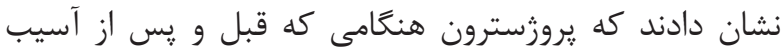

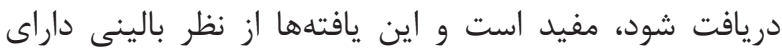

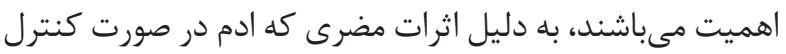

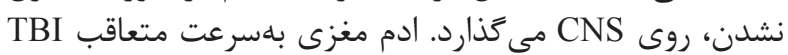

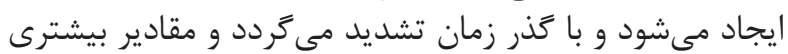

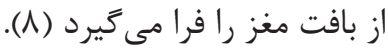

تحقيقات روى يرورزترون نشان داده است كه اين هورمون

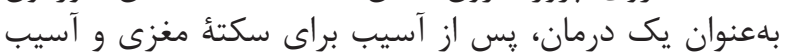

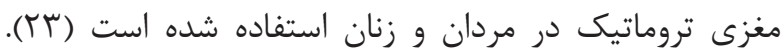

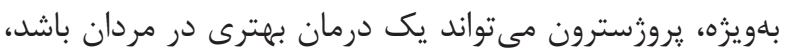

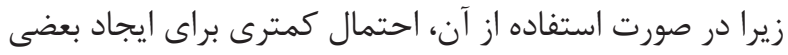

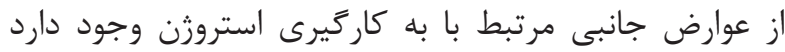

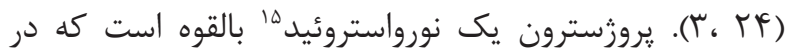

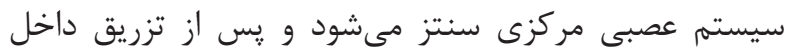

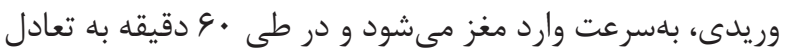

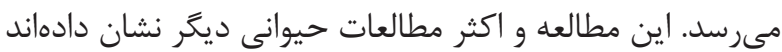

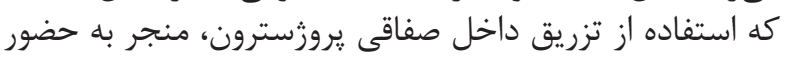

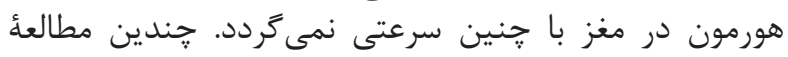

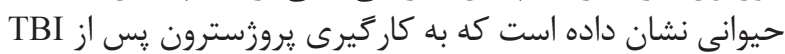

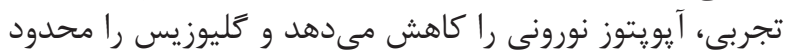

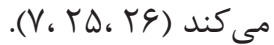

در واقع، يروزسترون مكانيسمهاى متعددى را درئ مرئ محفاظت

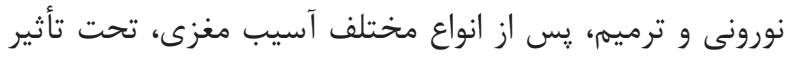

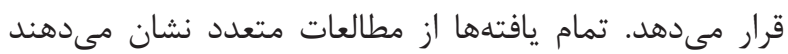

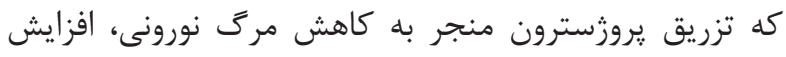

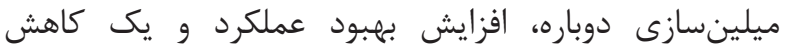
سراسرى در ادم مغزى مى كردد (TV) (T).

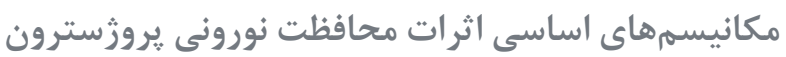

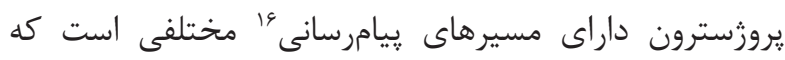

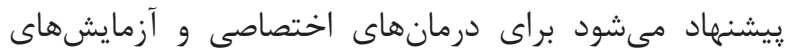

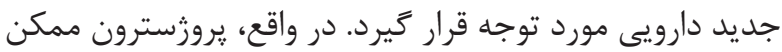

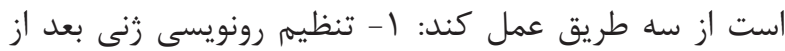

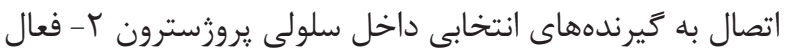

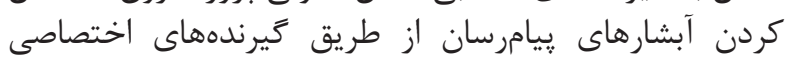

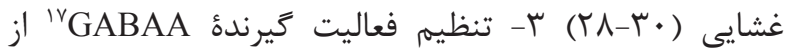

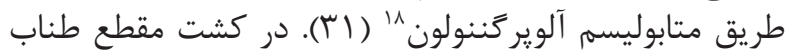

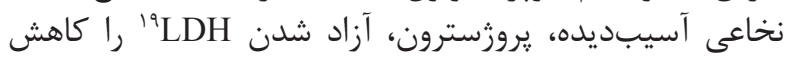

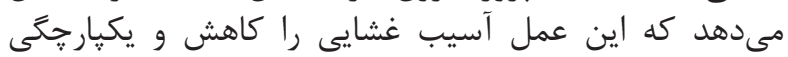
غشايى را افزايش مى دهد.

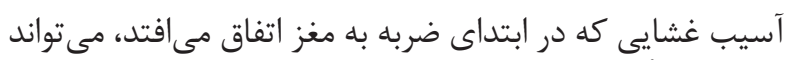
منجر به تأخير در ترميم و حتى مركى سلولى شود. از دست مست الفت

${ }^{22}$ Vasogenic
${ }^{23}$ Cytotoxic
${ }^{24}$ Aquaporins 4
${ }^{25}$ Vasopressin
${ }^{26}$ Neurogenic
${ }^{27}$ Blood brain barrier
${ }^{28}$ Matrix metalloproteinase




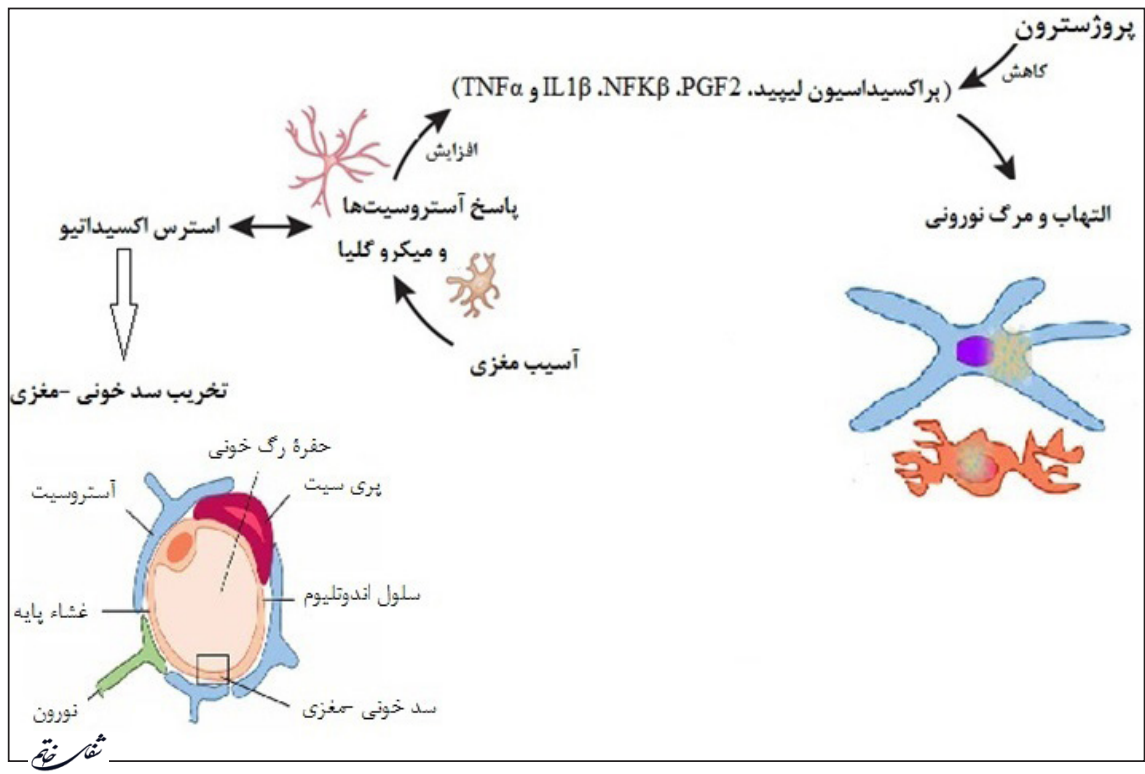

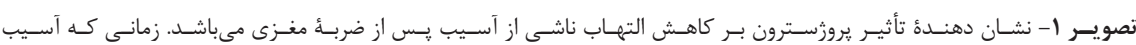

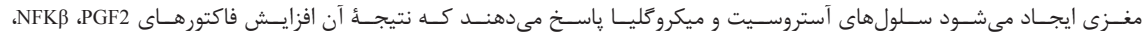

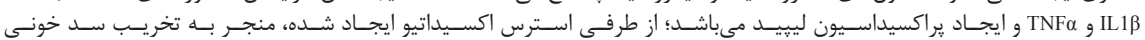

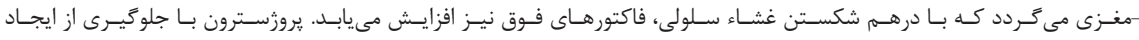

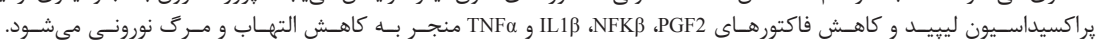

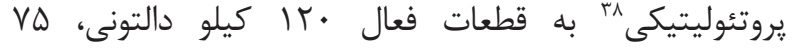

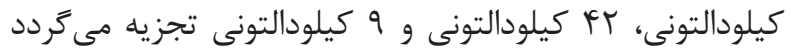

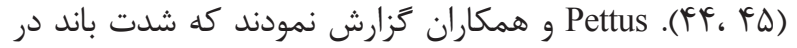

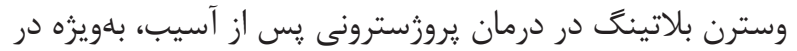

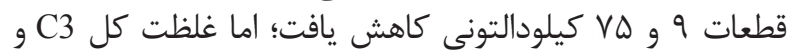

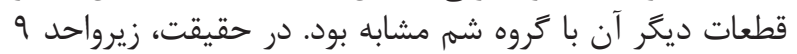

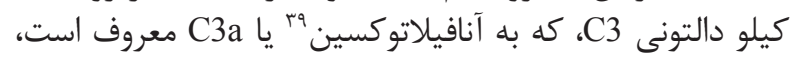

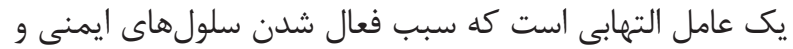

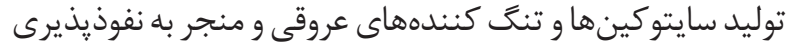

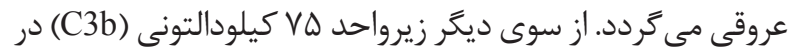

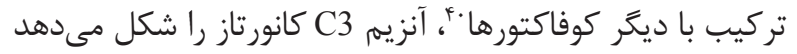

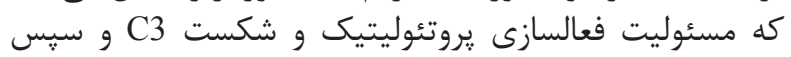
شكل كرفتن C3a را بر عهده دارد (9 ب).

در واقع مطالعات نشان دادهاند كه يروزسترون عامل تسهيل

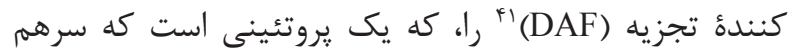

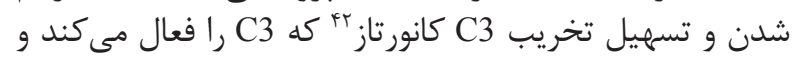

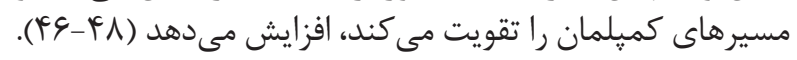
بنابراين فرضيهاي كه در اينجا توسط

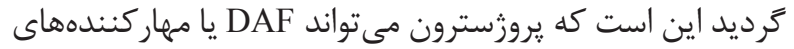

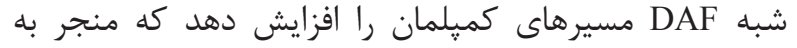

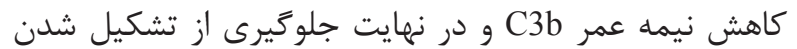

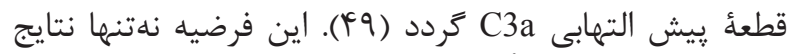

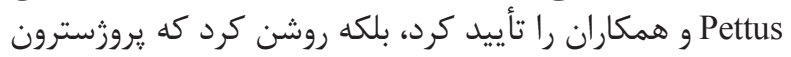
جطور فعاليتهاى ضدالتهابى را ميانجيكرى مى كند.

\footnotetext{
${ }^{29}$ Prostaglandin E2

${ }^{30}$ Complement component 3

${ }^{31}$ Cyclooxygenase-2

${ }^{32}$ Nuclear factor kappa B

${ }^{33}$ Tumor necrosis factor alpha

${ }^{34}$ Prostaglandins

${ }^{35}$ Prostacyclins
}

ديخر، مطالعات متعدد نشان داده است كه يروزسترون بر روى إئ

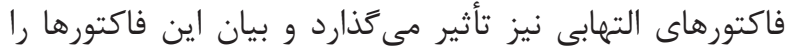

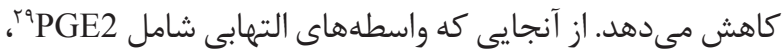
Cl C3

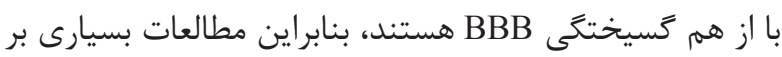

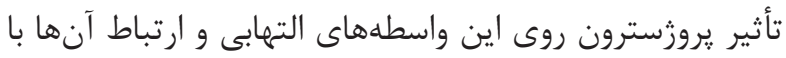

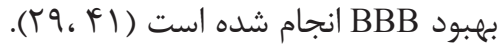

$$
\text { اثر يروزسترون بر PGE2 }
$$
در طى فرايند التهاب يست تروماتيك، محصولات متابوليك اسيد

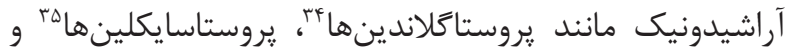

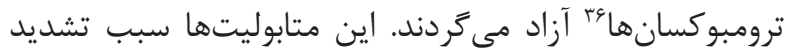

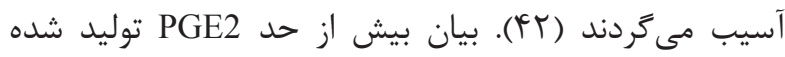

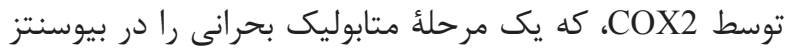

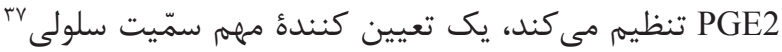

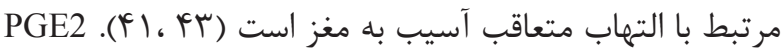

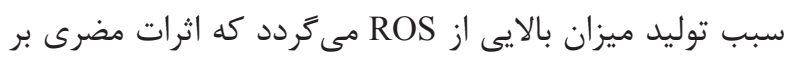

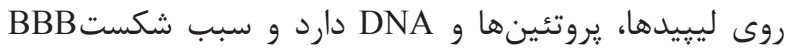

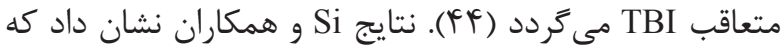

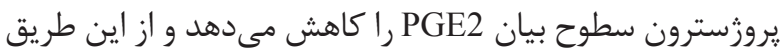

سبب كاهش سطح آسيب ايجاد شده مى گردد (تصوير ().

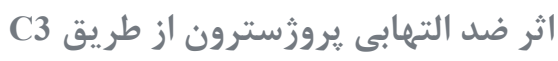

C3 يك يروتئين AD ا كيلودالتونى است كه به صورت
${ }^{36}$ Thromboxanes
${ }^{37}$ Cytotoxicity
${ }^{38}$ Proteolytic
${ }^{39}$ Anaphylatoxin
${ }^{40}$ Cofactors
${ }^{41}$ Decay-accelerating factor
${ }^{42}$ Conortaz 
مى كردد و بدين صورت رونويسى التهاب با واسطة NF-kB ، ماهش ) ماهش

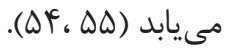

اثرات مهارى يروزسترون بر روى ادم مغزى

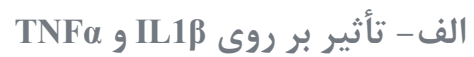

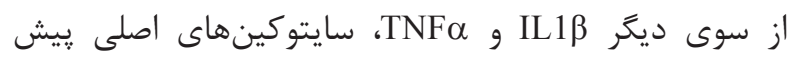

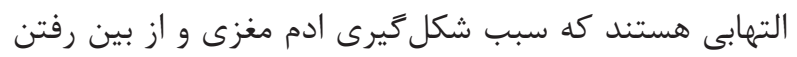

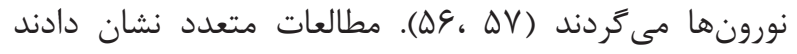

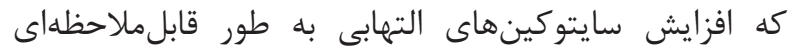

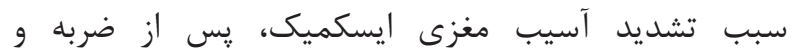

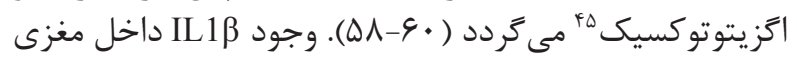

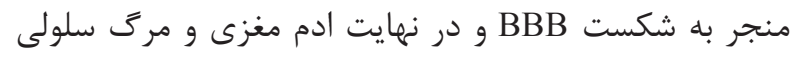
ثانويه مى گردد (

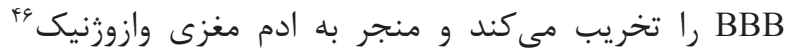

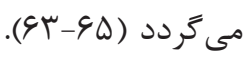

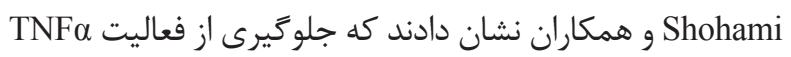

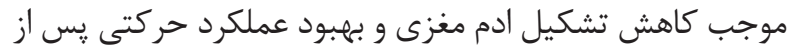
از آسي شود (98I

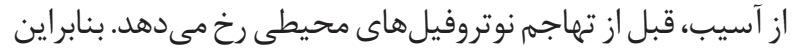

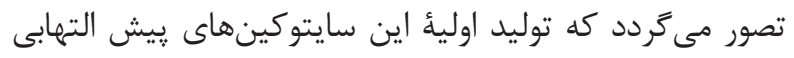

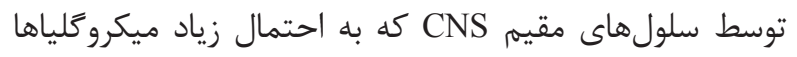

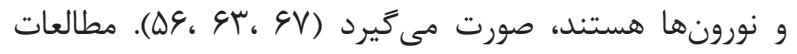

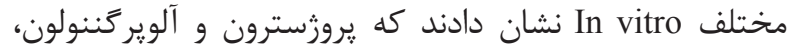

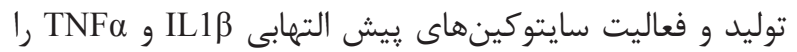

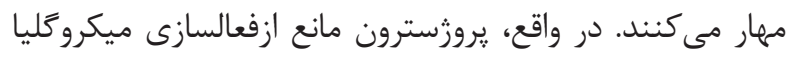

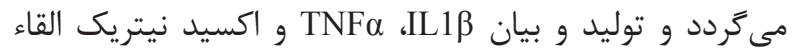

اثرات مهارى و تنظيم كاهشى يروزسترون بر روى NF-kB

$\operatorname{COX} 2$

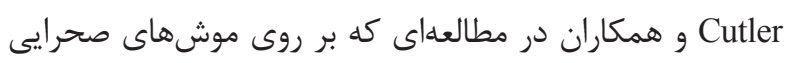

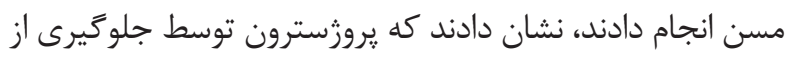

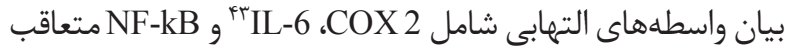

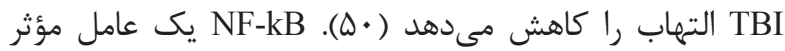

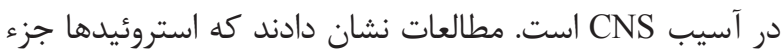

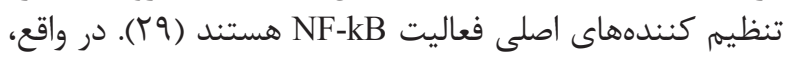

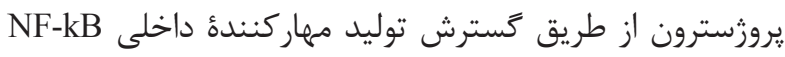
(IKB)

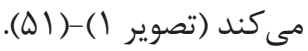

همجنين مطالعه روى اثرات ضد التهابى يروزسترون بر بر بافت

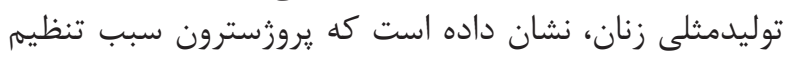

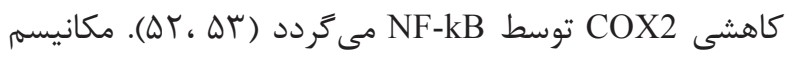

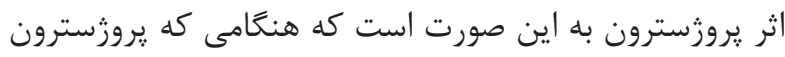

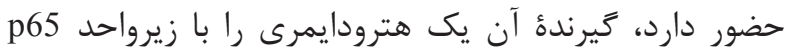

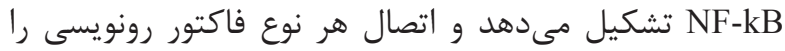

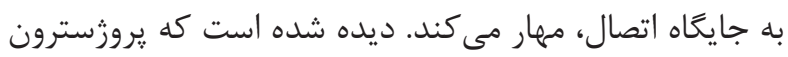

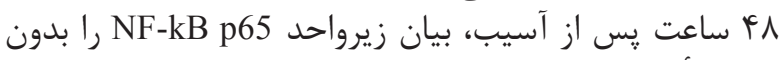

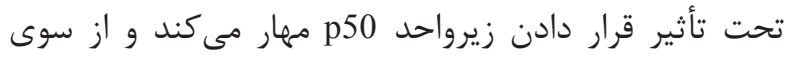

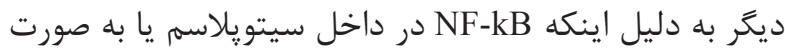

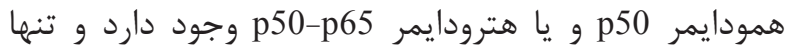

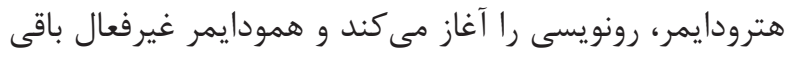

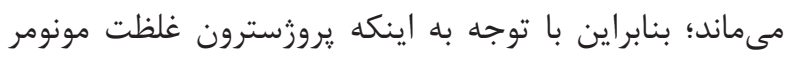

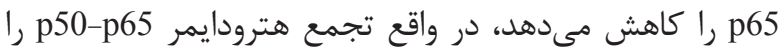

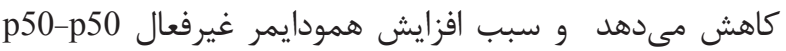

جدول ا- اثرات محافظت نورونى يروزسترون و كاهش ادم مغزى متعاقب TBI.

\begin{tabular}{|c|c|}
\hline توضيحات & كاهش سايتوكينهاى التهابى \\
\hline 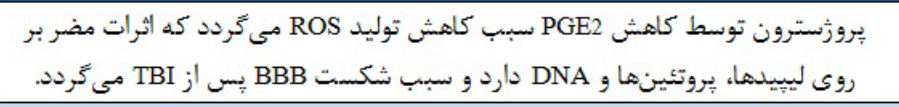 & كاهش PGE2 \\
\hline 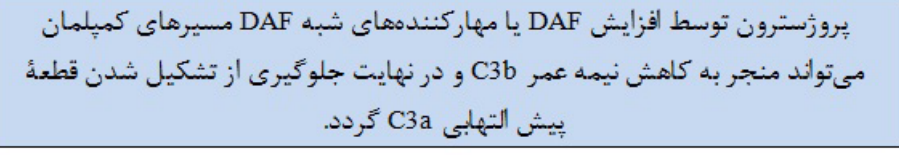 & 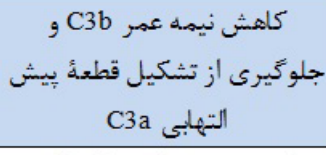 \\
\hline 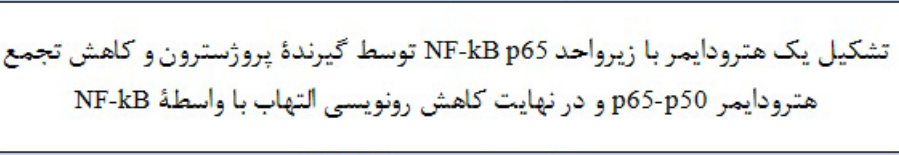 & 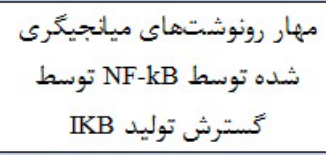 \\
\hline از طريق اثر بر مسير NF-kB & سبب تنظيم كامشى COX2 \\
\hline از طريق ممانعت از فعالسازى ميكروكليا & 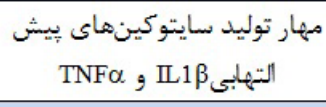 \\
\hline 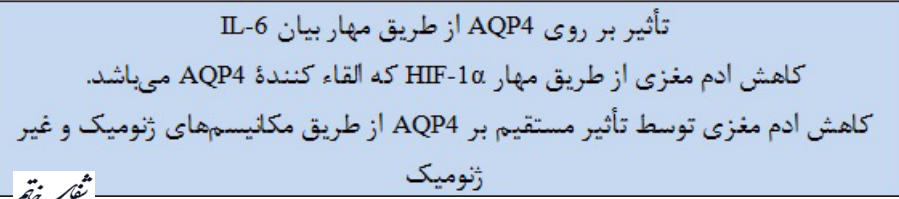 & AQP4 مهار بيان AQ \\
\hline
\end{tabular}

\footnotetext{
${ }^{43}$ Interleukin 6

${ }^{44}$ Inhibitor of kappa B

${ }^{45}$ Excitotoxicity

${ }^{46}$ Vasogenic
} 
اثرات وابسته به زمان :بروزسترون بر مهار سايتوكينهاى بيش التهابى

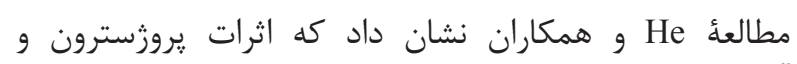

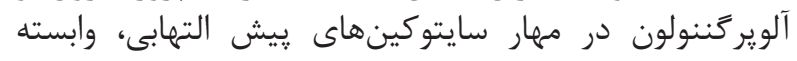

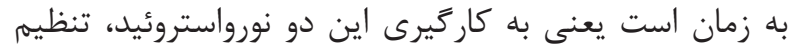

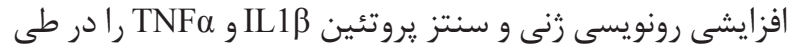

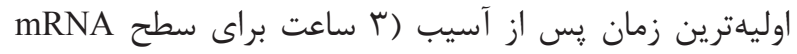

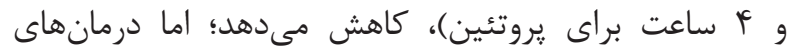

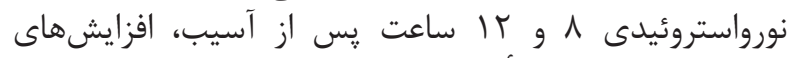

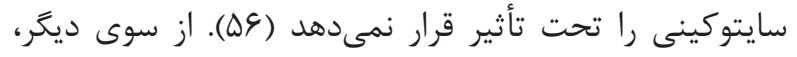

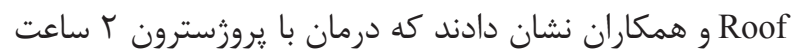

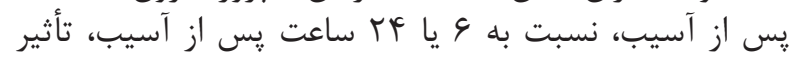

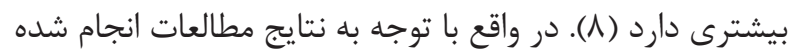

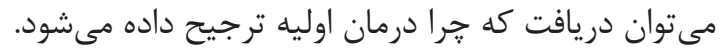
نتيجه كيرى

ادم مغزى يك افزايش مايع در داخل بافت مغزى، مى مباشد كه به به

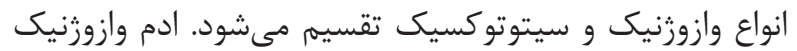

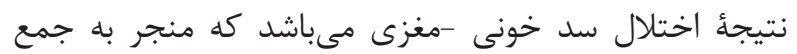

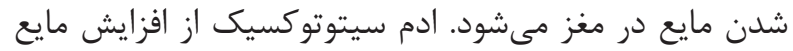

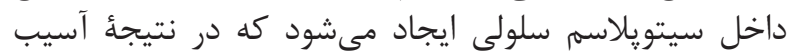

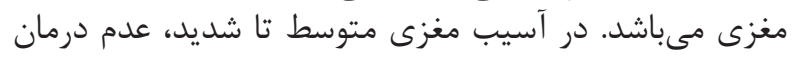

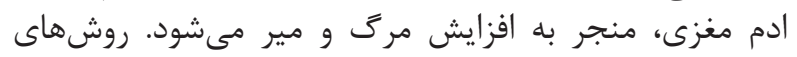

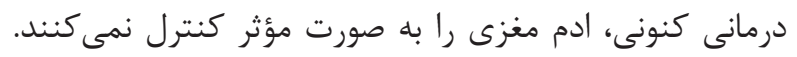

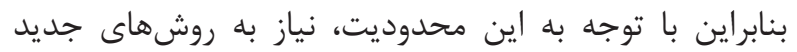

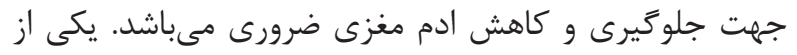
روشهاى نوين جهت كاهش ادم ادم مغزى استفاده از استروئيديدها

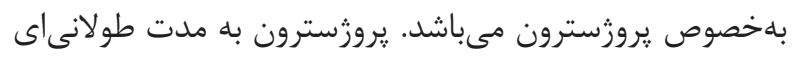

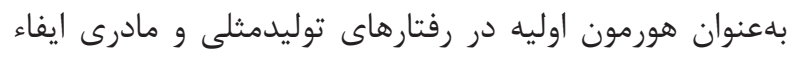

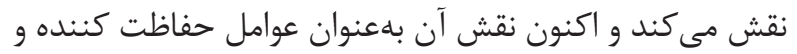

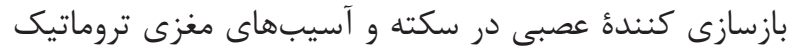

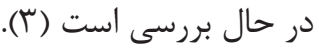

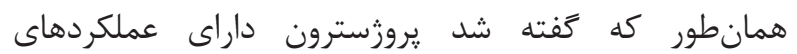

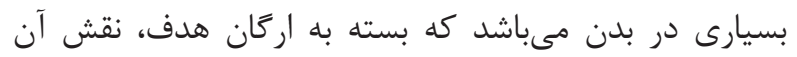

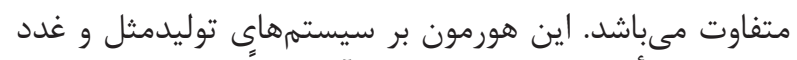

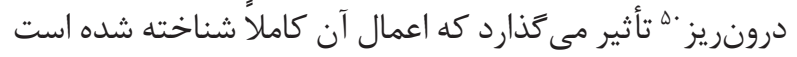

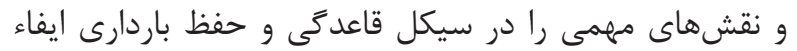

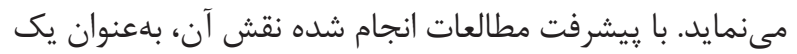

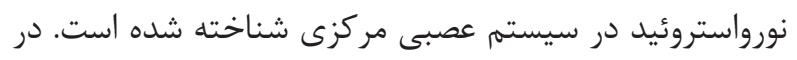

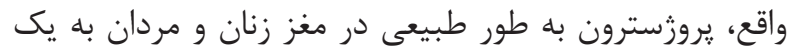

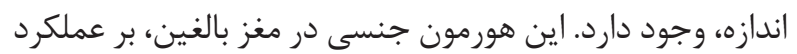

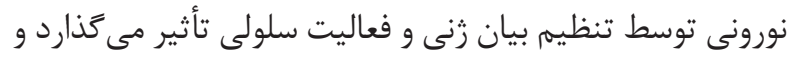

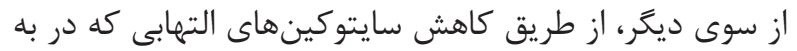

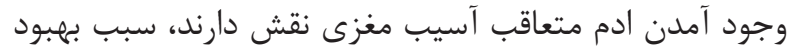

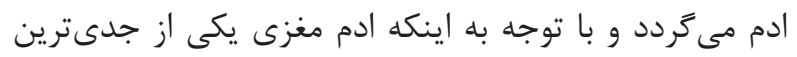

${ }^{47}$ Aquaporins

${ }^{48}$ Pathophysiology

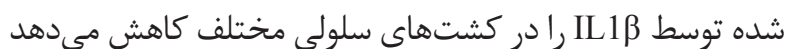

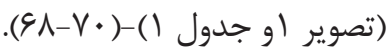
ب - تأثير بر روى Aquaporin و و

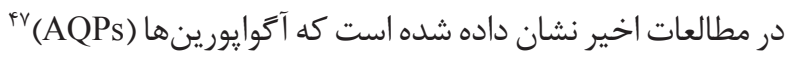

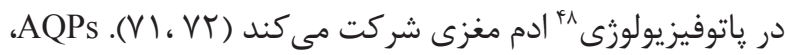

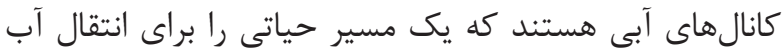

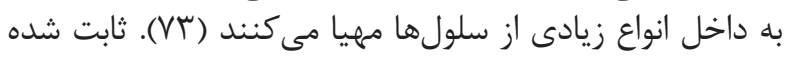

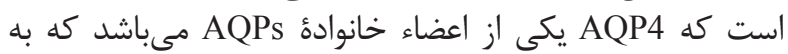

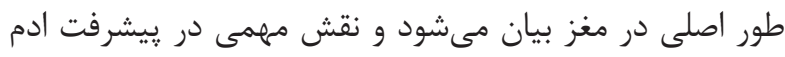

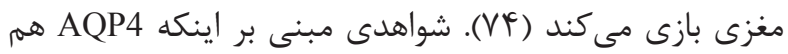

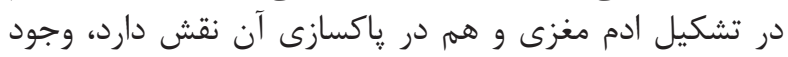

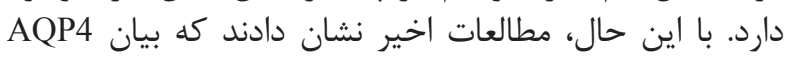

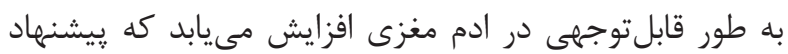

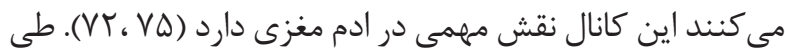

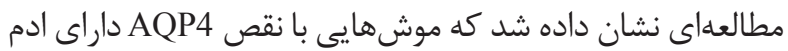

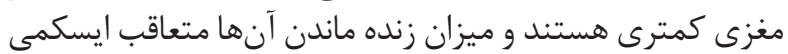

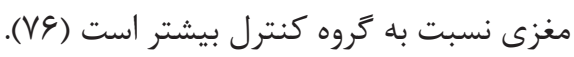

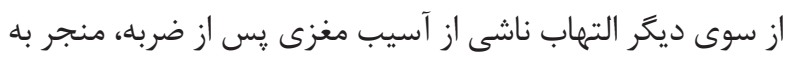

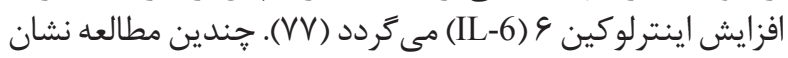

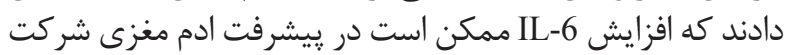

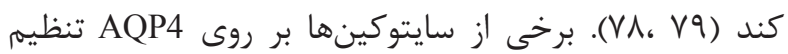

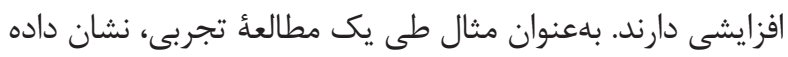

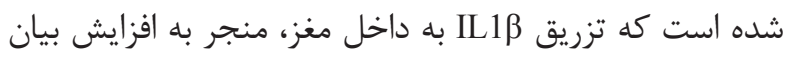

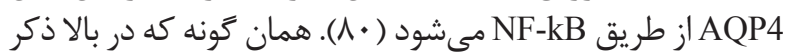

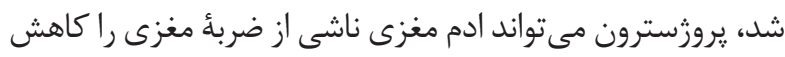

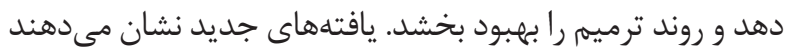

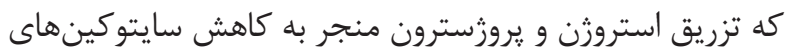

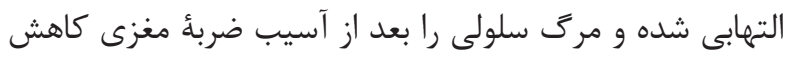

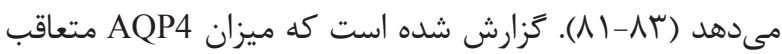

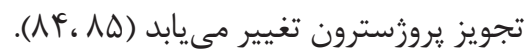

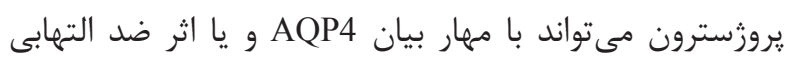

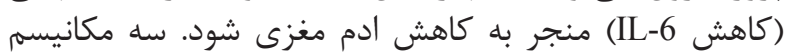

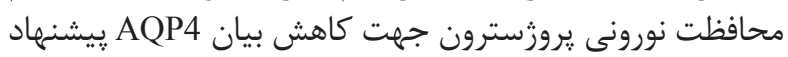

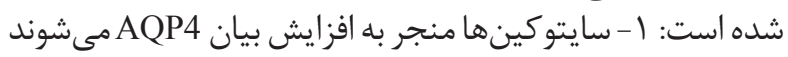

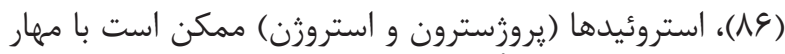

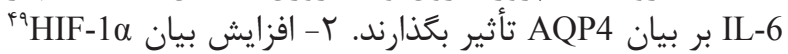

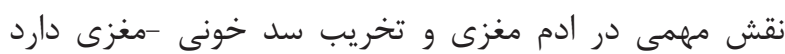

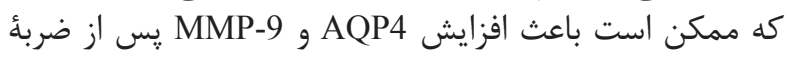

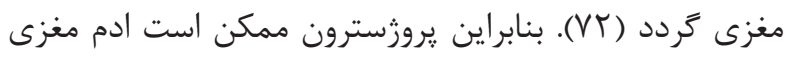

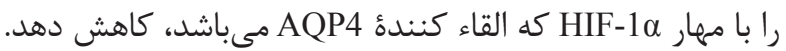

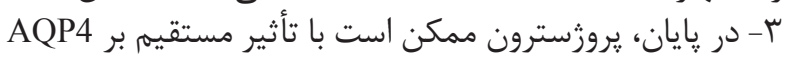

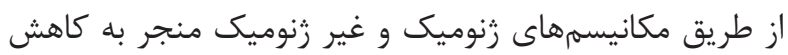

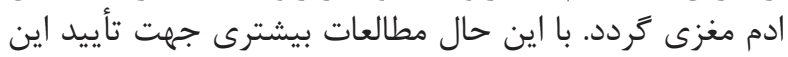

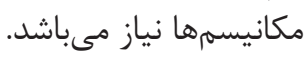

\footnotetext{
${ }^{49}$ Hypoxia-inducible factor 1 -alpha

${ }^{50}$ Endocrine
} 


$$
\begin{aligned}
& \text { آسيب مغزى يس از ضربه وجود ندارد. بنابراين، با توجه به } \\
& \text { توليد يروزسترون توسط سلولهاى مغزى و و وجود كيرندهنهاى }
\end{aligned}
$$

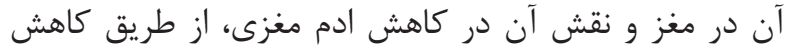

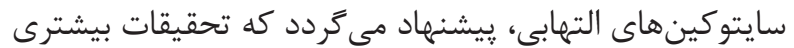

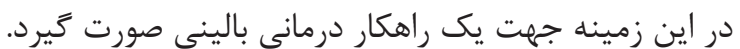

1. Ghadiri T, Sharifzadeh M, Khodagholi F, Modarres Mousavi SM, Hassanzadeh G, Zarrindast MR, et al. A novel traumatic brain injury model for induction of mild brain injury in rats. J Neurosci Methods. 2014; 233: $18-27$.

2. Nourzad Z, Khazali H, Ghadiri T, Modarres Mousavi M, Karimzadeh F, Eshaghabadi A, et al. Neuroprotective effects of concomitant use of erythropoietin and progesterone in traumatic brain injury. Shefaye Khatam. 2014; 2 (2): 1-12.

3. Stein DG. Brain damage, sex hormones and recovery: a new role for progesterone and estrogen? Trends Neurosci. 2001; 24(7): 386-91.

4. Wei J, Xiao GM. The neuroprotective effects of progesterone on traumatic brain injury: current status and future prospects. Acta Pharmacol Sin. 2013; 34(12): $1485-90$

5. Mohammadzadeh E. P15: The edema is one of the most significant side effect of accident. Shefaye Khatam. 2016; 3(S3): 42-42.

6. Roof RL, Duvdevani R, Stein DG. Gender influences outcome of brain injury: progesterone plays a protective role. Brain Res. 1993; 607(1): 333-6.

7. Pascual JL, Murcy MA, Li S, Gong W, Eisenstadt $\mathrm{R}$, Kumasaka $\mathrm{K}$, et al. Neuroprotective effects of progesterone in traumatic brain injury: blunted in vivo neutrophil activation at the blood-brain barrier. Am J Surg. 2013; 206(6): 840-6.

8. Roof RL, Duvdevani R, Heyburn JW, Stein DG. Progesterone rapidly decreases brain edema: treatment delayed up to 24 hours is still effective. Exp Neurol. 1996; 138(2): 246-51.

9. Hossmann KA. Treatment of experimental cerebral ischemia. J Cereb Blood Flow Metab. 1982; 2(3): 275-97.

10. Bremer AM, Yamada K, West CR. Ischemic cerebral edema in primates: effects of acetazolamide, phenytoin, sorbitol, dexamethasone, and methylprednisolone on

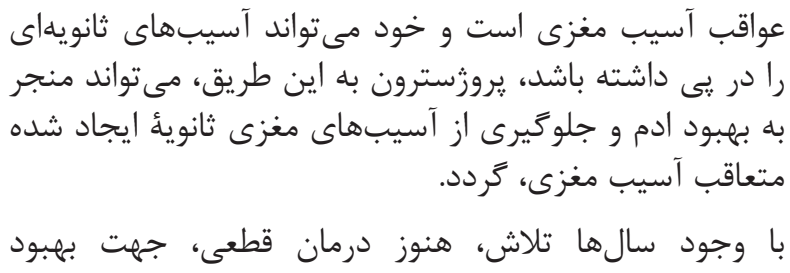

brain water and electrolytes. Neurosurgery. 1980; 6(2): 149-54.

11. Hall ED. Lipid antioxidants in acute central nervous system injury. Ann Emerg Med. 1993; 22(6): 1022-7.

12. Braughler JM, Hall ED. Lactate and pyruvate metabolism in injured cat spinal cord before and after a single large intravenous dose of methylprednisolone. J Neurosurg. 1983; 59(2): 256-61.

13. Braughler JM, Lainer MJ. The effects of large doses of methylprednisolone on neurologic recovery and survival in the Mongolian gerbil following three hours of unilateral carotid occlusion. Cent Nerv Syst Trauma. 1986; 3(2): 153-61.

14. Hall ED, Braughler JM. Acute effects of intravenous glucocorticoid pretreatment on the in vitro peroxidation of cat spinal cord tissue. Exp Neurol. 1981; 73(1): 3214.

15. Hall ED, Braughler JM. Effects of intravenous methylprednisolone on spinal cord lipid peroxidation and $(\mathrm{Na}++\mathrm{K}+)$-ATPase activity: Dose-response analysis during 1st hour after contusion injury in the cat. J Neurosurg. 1982; 57(2): 247-53.

16. Braughler JM, Hall ED. Uptake and elimination of methylprednisolone from contused cat spinal cord following intravenous injection of the sodium succinate ester. J Neurosurg. 1983; 58(4): 538-42.

17. Hall ED. Neuroprotective actions of glucocorticoid and nonglucocorticoid steroids in acute neuronal injury. Cell Mol Neurobiol. 1993; 13(4): 415-32.

18. Mohammadzadeh E, Sahab Negah S, Eshaghabadi A. P12: progesterone act as neuroprotective in traumatic brain injury. Shefaye Khatam. 2016; 3(S3): 39-39.

19. Xu FF, Sun S, Ho AS, Lee D, Kiang KM, Zhang $\mathrm{XQ}$, et al. Effects of progesterone vs. dexamethasone on brain oedema and inflammatory responses following experimental brain resection. Brain Inj. 2014; 28(12): 1594-601. 
20. Schumacher M, Robel P, Baulieu EE. Development and regeneration of the nervous system: a role for neurosteroids. Dev Neurosci. 1996; 18(1-2): 6-21.

21. Chen J, Chopp M, Li Y. Neuroprotective effects of progesterone after transient middle cerebral artery occlusion in rat. J Neurol Sci. 1999; 171(1): 24-30.

22. Mohammadzadeh E. P20: progesterone in tbi treatment. Shefaye Khatam. 2016; 3 (S3): 47-47.

23. Maghool F, Khaksari M, Asadi karam G. P10: effects of female gonadal hormones on neuromedin $\mathrm{s}$ and its receptor following experimental traumatic brain injury. Shefaye Khatam. 2015; 2 (S3): 60-60.

24. Soltani Z, Mofid B, Shahrokhi N, Khaksari M, Karamouzian S, Nakhaeei N, et al. P7: salutary effects of progesterone for traumatic brain injury. Shefaye Khatam. 2015; 2 (S3): 57-57.

25. Nourzad Z, Ghadiri T, Eshaghabadi A, Modarres Mousavi M. P58: neurological outcomes after traumatic brain injury affected by concomitant administration of progesterone and erythropoietin. Shefaye Khatam. 2015; 2 (S3): 108-108.

26. Nourzad Z, Ghadiri T, Modarres Mousavi M, Karimzadeh F, Eshaghabadi A, Hosseini Ravandi H, et al. P86: effect of concomitant use of erythropoietin and progesterone in traumatic brain injury. Shefaye Khatam. 2015; 2 (S3): 136-136.

27. Sahab Negah S, Khaksar Z, Modarres Mousavi M, Jahanbazi Jahan-Abad A, Eshaghabadi A, Hossini Ravandi H. P16: the role of astrocyte in traumatic brain injury. Shefaye Khatam. 2016; 3 (S3): 43-43.

28. Lösel RM, Falkenstein E, Feuring M, Schultz A, Tillmann HC, Rossol-Haseroth K, et al. Nongenomic steroid action: controversies, questions, and answers. Physiol Rev. 2003; 83(3): 965-1016.

29. Pettus EH, Wright DW, Stein DG, Hoffman SW. Progesterone treatment inhibits the inflammatory agents that accompany traumatic brain injury. Brain Res. 2005; 1049(1): 112-9.

30. Mani S. Signaling mechanisms in progesteroneneurotransmitter interactions. Neuroscience. 2006; 138(3): 773-81.

31. Belelli D, Lambert JJ. Neurosteroids: endogenous regulators of the GABA(A) receptor. Nat Rev Neurosci. 2005; 6(7): 565-75.

32. Ardeshiri A, Kelley M, Korner I, Hurn P, Herson
P. Mechanism of progesterone neuroprotection of rat cerebellar Purkinje cells following oxygen-glucose deprivation. Eur J Neurosci. 2006; 24(9): 2567-74.

33. Labombarda F, Ghoumari AM, Liere P, De Nicola AF, Schumacher M, Guennoun R. Neuroprotection by steroids after neurotrauma in organotypic spinal cord cultures: a key role for progesterone receptors and steroidal modulators of $\mathrm{GABA}(\mathrm{A})$ receptors. Neuropharmacology. 2013; 71: 46-55.

34. Chaplin ER, Free RG, Goldstein GW. Inhibition by steroids of the uptake of potassium by capillaries isolated from rat brain. Biochem Pharmacol. 1981; 30(3): 241-5.

35. Smith SL, Andrus PK, Zhang JR, Hall ED. Direct measurement of hydroxyl radicals, lipid peroxidation, and blood-brain barrier disruption following unilateral cortical impact head injury in the rat. J Neurotrauma. 1994; 11(4): 393-404.

36. Wolff JE, Laterra J, Goldstein GW. Steroid inhibition of neural micro vessel morphogenesis in vitro: receptor mediation and astroglial dependence. J Neurochem. 1992; 58(3): 1023-32.

37. Plum F, Alvord EC, Posner JB. Effect of steroids on experimental cerebral infarction. Arch Neurol. 1963; 9(6): 571-3.

38. Naderi V, Khaksari M, Maghool F. P6: neuroprotective effect of estrogen against brain edema and blood brain barrier disruption: roles of estrogen receptors $\alpha$ and $\beta$. Shefaye Khatam. 2015; 2(S3): 56-56.

39. Olson JJ, Poor MM, Beck DW. Methylprednisolone reduces the bulk flow of water across an in vitro bloodbrain barrier. Brain Res. 1988; 439(1): 259-65.

40. Attella MJ, Nattinville A, Stein DG. Hormonal state affects recovery from frontal cortex lesions in adult female rats. Behav Neural Biol. 1987; 48(3): 352-67.

41. Si D, Li J, Liu J, Wang X2, Wei Z, Tian Q, et al. Progesterone protects blood-brain barrier function and improves neurological outcome following traumatic brain injury in rats. Exp Ther Med. 2014; 8(3): 1010-4.

42. Knöferl MW, Diodato MD, Schwacha MG, Cioffi WG, Bland KI, Chaudry IH. Cyclooxygenase-2-mediated regulation of kupffer cell interleukin-6 production following trauma-hemorrhage and subsequent sepsis. Shock. 2001; 16(6): 479-83.

43. Buccellati C, Folco G, Sala A, Scelsi R, Masoero E, Poggi $\mathrm{P}$, et al. Inhibition of prostanoid synthesis protects against neuronal damage induced by focal ischemia in 


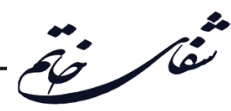

rat brain. Neurosci Lett. 1998; 257(3): 123-6.

44. Beaumont A, Marmarou A, Fatouros P, Corwin F. Secondary insults worsen blood brain barrier dysfunction assessed by MRI in cerebral contusion. Acta Neurochir Suppl. 2002; 81: 217-9.

45. Keeling K, Hicks R, Mahesh J, Billings B, Kotwal G. Local neutrophil influx following lateral fluidpercussion brain injury in rats is associated with accumulation of complement activation fragments of the third component (C3) of the complement system. J Neuroimmunol. 2000; 105(1): 20-30.

46. Brodbeck WG, Mold C, Atkinson JP, Medof ME. Cooperation between decay-accelerating factor and membrane cofactor protein in protecting cells from autologous complement attack. T J Immunol. 2000; 165(7): 3999-4006.

47. Rinder CS, Rinder HM, Johnson K, Smith M, Lee DL, Tracey J, et al. Role of C3 cleavage in monocyte activation during extracorporeal circulation. Circulation. 1999; 100(5): 553-8.

48. van Beek J, van Meurs M, t Hart BA, Brok HP, Neal JW, Chatagner A, et al. Decay-accelerating factor (CD55) is expressed by neurons in response to chronic but not acute autoimmune central nervous system inflammation associated with complement activation. J Immunol. 2005; 174(4): 2353-65.

49. Kaul A, Nagamani M, Nowicki B. Decreased expression of endometrial decay accelerating factor (DAF), a complement regulatory protein, in patients with luteal phase defect. Am J Reprod Immunol. 1995; 34(4): 236-40.

50. Cutler SM, Cekic M, Miller DM, Wali B, VanLandingham JW, Stein DG. Progesterone improves acute recovery after traumatic brain injury in the aged rat. J Neurotrauma. 2007; 24(9): 1475-86.

51. Wissink S, Van Heerde E, Van der Burg B, Van der Saag P. A dual mechanism mediates repression of NF$\kappa \mathrm{B}$ activity by glucocorticoids. Mol Endocrinol. 1998; 12(3): 355-63.

52. Allport V, Slater D, Newton R, Bennett P. NF-кB and AP-1 are required for cyclo-oxygenase 2 gene expression in amnion epithelial cell line (WISH). Mol Hum Reprod. 2000; 6(6): 561-5.

53. Allport V, Pieber D, Slater D, Newton R, White J, Bennett P. Human labour is associated with nuclear factor- $\kappa \mathrm{B}$ activity which mediates cyclo-oxygenase-2 expression and is involved with the 'functional progesterone withdrawal'. Mol Hum Reprod. 2001; 7(6): 581-6.

54. Ghosh S, Karin M. Missing pieces in the NF-кB puzzle. Cell. 2002; 109(2): S81-S96.

55. Gu Z, Cain L, Werrbach-Perez K, Perez-Polo JR. Differential alterations of NF- $\kappa B$ to oxidative stress in primary basal forebrain cultures. Int J Dev Neurosci. 2000; 18(2): 185-92.

56. He J, Evans CO, Hoffman SW, Oyesiku NM, Stein DG. Progesterone and allopregnanolone reduce inflammatory cytokines after traumatic brain injury. Exp Neurol. 2004; 189(2): 404-12.

57. Sahab Negah S, Khaksar Z, Jahanbazi Jahan-Abad A, Eshaghabadi A, Modarres Mousavi M, Hossini Ravandi H. P21: is interleukin-1 beta correlated with intra cranial pressure after traumatic brain injury? Shefaye Khatam. 2016; 3 (S3): 48-48.

58. Lawrence CB, Allan SM, Rothwell NJ. Interleukin$1 \beta$ and the interleukin-1 receptor antagonist act in the striatum to modify excitotoxic brain damage in the rat. Eur J Neurosci. 1998; 10(3): 1188-95.

59. Loddick SA, Rothwell NJ. Neuroprotective effects of human recombinant interleukin-1 receptor antagonist in focal cerebral ischaemia in the rat. J Cereb Blood Flow Metab. 1996; 16(5): 932-40.

60. Yamasaki Y, Matsuura N, Shozuhara H, Onodera H, Itoyama Y, Kogure K. Interleukin-1 as a pathogenetic mediator of ischemic brain damage in rats. Stroke. 1995; 26(4): 676-81.

61. Holmin S, Mathiesen T. Intracerebral administration of interleukin-1 $\beta$ and induction of inflammation, apoptosis, and vasogenic edema. J Neurosurg. 2000; 92(1): 108-20.

62. Touzani O, Boutin H, Chuquet J, Rothwell N. Potential mechanisms of interleukin-1 involvement in cerebral ischaemia. J Neuroimmunol. 1999; 100(1): 203-15.

63. Knoblach SM, Fan L, Faden AI. Early neuronal expression of tumor necrosis factor- $\alpha$ after experimental brain injury contributes to neurological impairment. J Neuroimmunol. 1999; 95(1): 115-25.

64. Megyeri P, Ábrahám CS, Temesvári P, Kovács J, Vas T, Speer CP. Recombinant human tumor necrosis factor 
$\alpha$ constricts pial arterioles and increases blood-brain barrier permeability in newborn piglets. Neurosci Lett. 1992; 148(1): 137-40.

65. Stolpen AH, Guinan EC, Fiers W, Pober JS. Recombinant tumor necrosis factor and immune interferon act singly and in combination to reorganize human vascular endothelial cell monolayers. Am J Pathol. 1986; 123(1): 16-24.

66. Shohami E, Bass R, Wallach D, Yamin A, Gallily R. Inhibition of tumor necrosis factor alpha (TNF $\alpha)$ activity in rat brain is associated with cerebroprotection after closed head injury. J Cereb Blood Flow Metab. 1996; 16(3): 378-84.

67. Fan L, Young PR, Barone FC, Feuerstein GZ, Smith $\mathrm{DH}$, McIntosh TK. Experimental brain injury induces expression of interleukin-1 $\beta$ mRNA in the rat brain. Brain Res Mol Brain Res. 1995; 30(1): 125-30.

68. Drew PD, Chavis JA. Female sex steroids: effects upon microglial cell activation. J Neuroimmunol. 2000; 111(1): 77-85.

69. Polan ML, Loukides J, Nelson P, Carding S, Diamond M, Walsh A, et al. progesterone and estradiol modulate interleukin-1 $\beta$ messenger ribonucleic acid levels in cultured human peripheral monocytes. J Clin Endocrinol Metab. 1989; 69(6): 1200-6.

70. Salzman A, Linn S, Szabo C. Progesterone inhibits inducible nitric oxide synthase mRNA expression in human intestinal epithelial cells. Int J Mol Med. 2000; 6(2): 209-25.

71. Papadopoulos MC, Verkman A. Potential utility of aquaporin modulators for therapy of brain disorders. Prog Brain Res. 2008; 170: 589-601.

72. Higashida T, Kreipke CW, Rafols JA, Peng C, Schafer S, Schafer P, et al. The role of hypoxia-inducible factor-1 $\alpha$, aquaporin-4, and matrix metalloproteinase- 9 in blood-brain barrier disruption and brain edema after traumatic brain injury: laboratory investigation. $\mathrm{J}$ Neurosurg. 2011; 114(1): 92-101.

73. Agre P, King LS, Yasui M, Guggino WB, Ottersen OP, Fujiyoshi Y, et al. Aquaporin water channels-from atomic structure to clinical medicine. J Physiol. 2002; 542(Pt 1): 3-16.

74. Tait MJ, Saadoun S, Bell BA, Papadopoulos MC. Water movements in the brain: role of aquaporins. Trends Neurosci. 2008; 31(1): 37-43.

75. Sun MC, Honey CR, Berk C, Wong NL, Tsui JK.
Regulation of aquaporin- 4 in a traumatic brain injury model in rats. J Neurosurg. 2003; 98(3): 565-9.

76. Bloch O, Manley GT. The role of aquaporin-4 in cerebral water transport and edema. Neurosurg Focus. 2007; 22(5): 1-7.

77. Hillman J, Åneman O, Persson M, Andersson C, Dabrosin C, Mellergård P. Variations in the response of interleukins in neurosurgical intensive care patients monitored using intracerebral microdialysis. J Neurosurg. 2007; 106(5): 820-5.

78. Tomás-Camardiel M, Venero J, Herrera A, De Pablos R, Pintor-Toro JA, Machado A, et al. Bloodbrain barrier disruption highly induces aquaporin-4 mRNA and protein in perivascular and parenchymal astrocytes: Protective effect by estradiol treatment in ovariectomized animals. J Neurosci Res. 2005; 80(2): 235-46.

79. Vajtr D, Benada O, Kukacka J, Prusa R, Houstava L, Toupalik P, et al. Correlation of ultrastructural changes of endothelial cells and astrocytes occurring during blood brain barrier damage after traumatic brain injury with biochemical markers of blood brain barrier leakage and inflammatory response. Physiol Res. 2009; 58(2): 263-8.

80. Ito H, Yamamoto N, Arima H, Hirate H, Morishima T, Umenishi $F$, et al. Interleukin- $1 \beta$ induces the expression of aquaporin- 4 through a nuclear factor- $\kappa \mathrm{B}$ pathway in rat astrocytes. J Neurochem. 2006; 99(1): 107-18.

81. Djebaili M, Guo Q, Pettus EH, Hoffman SW, Stein DG. The neurosteroids progesterone and allopregnanolone reduce cell death, gliosis, and functional deficits after traumatic brain injury in rats. $\mathrm{J}$ Neurotrauma. 2005; 22(1): 106-18.

82. Gibson CL, Murphy SP. Progesterone enhances functional recovery after middle cerebral artery occlusion in male mice. J Cereb Blood Flow Metab. 2004; 24(7): 805-13.

83. Shahrokhi N, Khaksari M, Soltani Z, Mahmoodi M, Nakhaee N. Effect of sex steroid hormones on brain edema, intracranial pressure, and neurologic outcomes after traumatic brain injury. Can J Physiol Pharmacol. 2010; 88(4): 414-21.

84. Guo Q, Sayeed I, Baronne LM, Hoffman SW, Guennoun R, Stein DG. Progesterone administration modulates AQP4 expression and edema after traumatic brain injury in male rats. Exp Neurol. 2006; 198(2): 46978.

85. Shin JA, Choi JH, Choi YH, Park EM. Conserved 


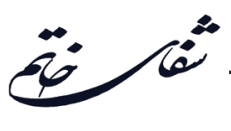

aquaporin 4 levels associated with reduction of brain edema are mediated by estrogen in the ischemic brain after experimental stroke. Biochim Biophys Acta. 2011; 1812(9): 1154-63.
86. Ding Z, Zhang J, Xu J, Sheng G, Huang G. Propofol administration modulates AQP-4 expression and brain edema after traumatic brain injury. Cell Biochem Biophys. 2013; 67(2): 615-22. 